\title{
DETERMINAÇÃO E ANÁLISE DE COEFICIENTE DE DESCARGA DE UM TUBO DE VENTURI COM AUXÍLIO DE UM SENSOR DE DIFERENÇA DE PRESSÃO MPX 5050
}

\section{DETERMINATION AND ANALYSIS OF THE COEFFICIENT OF DISCHARGE OF A VENTURI TUBE WITH THE AID OF A MPX 5050 DIFFERENTIAL PRESSURE SENSOR}

\author{
G. G. BARROS ${ }^{1, *}$ e H. M. P. ROSA ${ }^{1}$
}

${ }^{1}$ Universidade Federal de Viçosa, Departamento de Engenharia de Produção e Mecânica, Viçosa, Minas Gerais, Brasil

${ }^{*}$ Corresponding author. Departamento de Engenharia de Produção e Mecânica, Universidade Federal de Viçosa, Viçosa, Minas Gerais, Brasil, Fone: +55 31 3899-4968.

Endereço de E-mail: gabriel.garnica@ufv.br (G. G. Barros)

\begin{tabular}{l} 
A R T I C L E I N F O \\
\hline Article history: \\
Received 2018-12-20 \\
Accepted 2018-12-20 \\
Available online 2018-12-20 \\
pa lavras ch a ve \\
Venturi \\
Vazão \\
Sensor \\
Pressão \\
Descarga \\
\\
$k$ e y w o rds \\
Venturi \\
Flow \\
Sensor \\
Pressure \\
Discharge \\
\hline
\end{tabular}

\begin{abstract}
A B S T R A C T
This paper analyzes the use of a MPX5050 sensor in the determination of the discharge coefficient of a Venturi tube. In order to carry out the study, the discharge coefficient was manually measured with aid of a U-tube manometer, thus comparative analysis with the results obtained with the sensor was possible. The results showed that the coefficient determined by the sensor had an error of plus or minus five percent over the reference. The result confers to the sensor acceptable reliability, for applications of precision proportional to the error found, as well as allow further use in the automation of fluid-mechanical systems associated with one or more Venturi tubes and in the use for the determination of the coefficient of other devices.
\end{abstract}

R E S U M O

Este artigo analisa a utilização de um sensor de diferença de pressão do tipo MPX 5050 na determinação do coeficiente de descarga de um tubo de Venturi. Para realizar o estudo foi determinado o coeficiente de descarga manualmente com o auxílio de um manômetro em $U$, assim tornando possível uma análise comparativa com os resultados obtidos com o sensor. Os resultados mostraram que o coeficiente determinado pelo sensor teve um erro de mais ou menos cinco por cento em relação a referência. $O$ resultado confere ao sensor confiabilidade aceitável, para aplicações de precisão proporcional ao erro encontrado, assim abrindo espaço para o uso na automatização de sistemas fluidomecânicos associados a um ou mais tubos de Venturi e no uso para a determinação do coeficiente de outros dispositivos. 


\section{N O M E N C L A T U R A}

$D=$ Diâmetro maior do Venturi $(m)$

$d=$ Diâmetro menor do Venturi $(m)$

$q_{m}=$ Vazão mássica $(\mathrm{kg} / \mathrm{s})$

$\Delta h=$ Diferença de altura no manômetro $(m)$

$\Delta P=$ Diferença de pressão $(\mathrm{Pa})$

$\rho=$ Densidade da água $\left(\mathrm{kg} / \mathrm{m}^{3}\right)$

\section{INTRODUCÃO}

Este tubo de Venturi é um componente de circuitos hidráulicos utilizado para medir vazão de fluídos em tubos através da leitura da diferença de pressão entre duas seções do próprio Venturi, aplicada à equação de Bernoulli. É amplamente utilizado na indústria, principalmente na petroquímica, para o monitoramento de vazão de gás em alta pressão (Dehkordi et al., 2017), devido às sua simplicidade, alta confiabilidade e capacidade de gerar bons resultados mesmo em fluidos com duas fases (Li e Mickan, 2015). Além de seus usos clássicos na indústria, novos estudos têm levado sua aplicação a usos cada vez mais complexos como sua utilização em análise da respiração humana e consumo calórico (Titheradge e Robergs, 2018).
Para que seja utilizado, o Venturi precisa ter seu coeficiente de descarga analisado e descrito em relação a uma faixa de número de Reynolds, pois esse valor faz uma correção na equação de Bernoulli permitindo a leitura correta da vazão.

Definir a curva do coeficiente de descarga é uma tarefa laborosa, feita com o auxílio de um manômetro em U. Dessa forma, torna-se interessante o emprego de sensores para realizar esse ofício. Entretanto, para que o uso intensivo de sensores seja feito é necessário que a curva do coeficiente resultante de suas coletas seja semelhante à correta. Portanto, para garantir essa semelhança é necessário uma análise comparativa entre os dados coletados e a literatura.

\section{OBJETIVOS}

Este trabalho tem como objetivo analisar e comparar a curva do coeficiente de descarga de um mesmo tubo de Venturi feita manualmente utilizando um manômetro em $\mathrm{U}$, e uma curva feita através de coletas de um sensor de diferença de pressão MPX 5050, realizada com o auxílio de uma placa de Arduino Uno®, assim determinando a pertinência dessa associação.

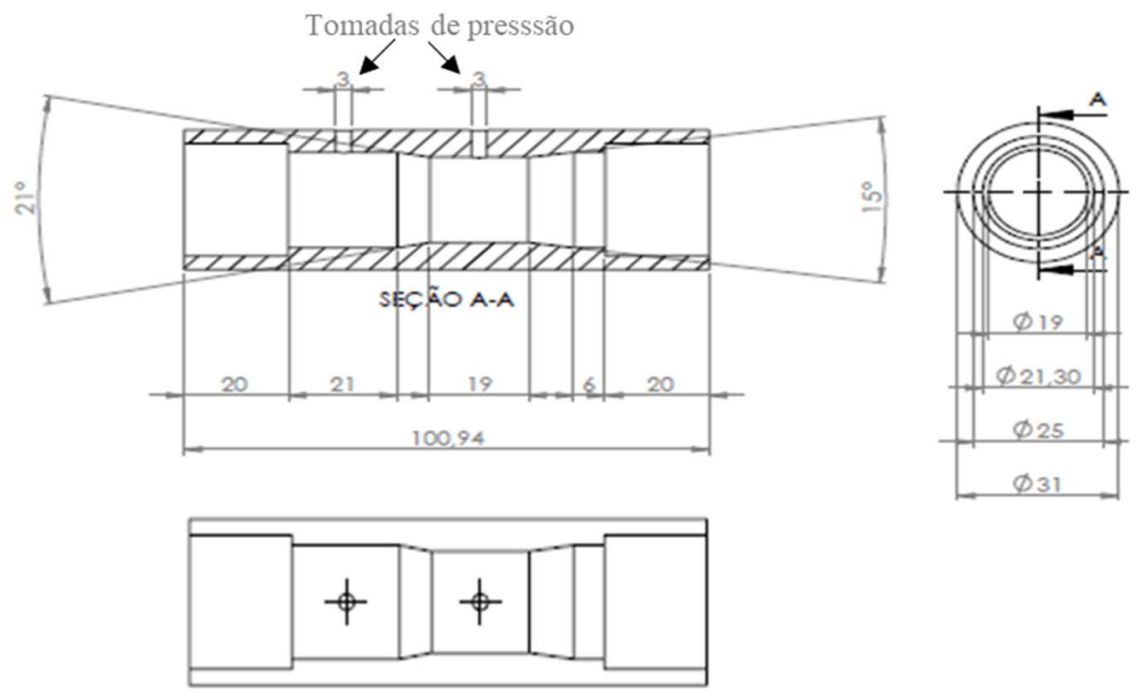

Figura 1 - Dimesões do tubo de Venturi, cotas em milímetros.

\section{MATERIAIS E MÉTODOS}

O tubo de Venturi utilizado, Figura 1, foi feito sob dimensões dentro dos limites estabelecidos segundo a ISO 5167. Sobre os pontos de tomada de pressão foram postos adaptadores para que tubos fossem associados, indo para o sensor de pressão ou sendo postos na vertical junto a uma superfície milimetrada formando um manômetro em U (Figura 2).

O sistema fluido-mecânico ao qual o Venturi participava era composto por uma bomba de $1 / 2 \mathrm{cv}$, uma válvula esfera e um reservatório ligado a entrada da bomba. Toda a tubulação do conjunto era de tubos de PVC de $3 / 4$ de polegada e a altura entre a bomba e o tubo era de aproximadamente $1,2 \mathrm{~m}$ e a distância entre o centro do reservatório e a bomba de aproximadamente $2,4 \mathrm{~m}$.

Para determinar o coeficiente de descarga para um valor de vazão é necessário determinar a vazão real e a vazão teórica de forma que a razão entre a real e a teórica é o próprio coeficiente. A determinação da vazão real é realizada manualmente através da aquisição de um volume de água com um recipiente calibrado associado a coleta do tempo decorrido utilizando um cronômetro. Por outro lado, a determinação da vazão teórica é feita com o uso da equação de Bernoulli adaptada para o objetivo de determinar a vazão mássica, conforme a equação 1 . 


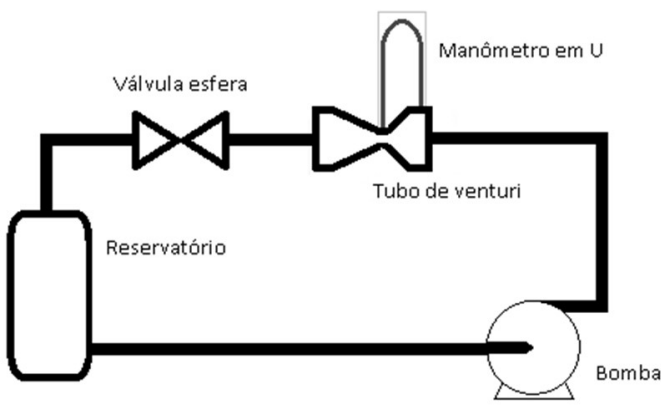

Figura 2 - Montagem com manômetro em U.

A diferença de pressão no manômetro em U é dada por $\Delta \mathrm{P}=\rho g \Delta \mathrm{h}$. A variação da vazão foi feita utilizando a válvula de esfera. Para cada condição de abertura da válvula foram feitas cinco coletas de medidas. As condições de abertura variaram entre totalmente aberta e $50 \%$.

$$
q_{m}=\frac{1}{\sqrt{1-\left(\frac{d}{D}\right)^{2}}} \frac{\pi d^{2}}{4} \sqrt{2 \rho \Delta P}
$$

Para a determinação da vazão teórica com o auxílio do sensor de diferença de pressão foi inicialmente feita a associação do sensor MPX 5050 (Figura 3) a uma placa de microcontrolador Arduino Uno ${ }^{\circledR}$ ligada a um computador. A conexão entre sensor e microcontrolador foi feita segundo as especificações do manual da Farnell.

O sensor MPX 5050 é do tipo piezo-resistivo e utiliza uma membrana de silicone (Figura 4). O sensor mede a pressão diferencial até $50 \mathrm{kPa}$. É alimentado por uma tensão de $5 \mathrm{~V}$, e a tensão de saída é proporcional à pressão. $\mathrm{O}$ gráfico da Figura 5 mostra a relação entre a tensão de saída e a pressão.
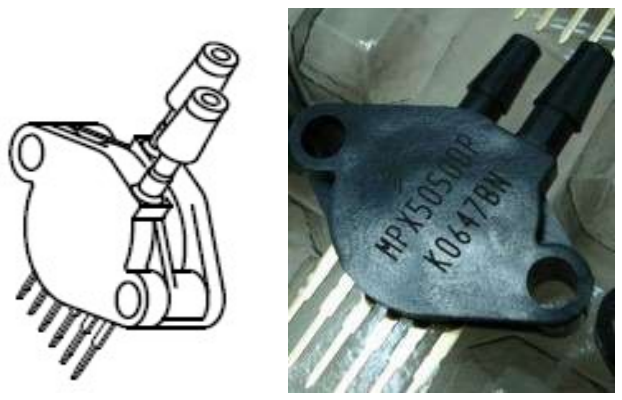

Figura 3 - Sensor MPX5050.

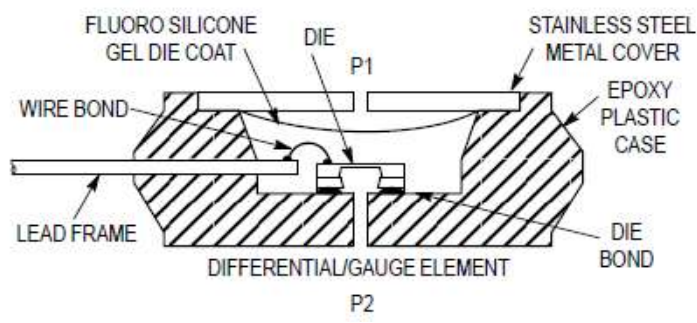

Figura 4 - Seção transversal do sensor MPX5050.

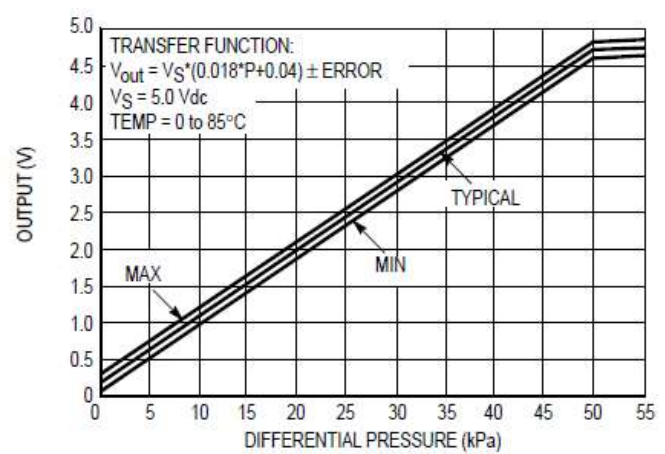

Figura 5 - Curva característica do sensor MPX5050.

Utilizando a relação extraída da curva característica (Figura 5) foi elaborado um código na linguagem do microcontrolador para a leitura e calibração do sensor. $\mathrm{O}$ código continha também comandos para definir a taxa de coleta de dados e o respectivo tempo. Para os ensaios foram aferidas duas coletas por segundo num tempo de 70 segundos, totalizando 140 medidas de pressão para cada conjunto de coletas. Foram realizados três conjuntos de coletas para cada uma das posições de abertura da válvula de esfera.

\section{RESULTADOS E DISCUSSÃO}

Os coeficientes encontrados ficaram dentro da faixa esperada segundo Reader-Harris (2015). A dispersão da curva do coeficiente de descarga feito com o auxílio do sensor foi aproximadamente inferior a 5\% em relação a curva feita com o manômetro em U para qualquer ponto na faixa analisada, como pode ser visto observando as Figuras 6 e 7 . Cada ponto nos gráficos representa a média do coeficiente de descarga encontrado para cada coleta.

A dispersão de dados do sensor também foi avaliada e $95 \%$ dos dados de cada coleta flutuavam em no máximo $5 \%$ da média das coletas obtidas. O formato das curvas dos gráficos e os valores dos coeficientes obtidos estão de acordo com a teoria para medidores Venturi, e com dados de ensaios disponíveis na literatura acadêmica e em catálogos de fabricante.

No manual do fabricante, do sensor MPX 5050, está descrito que o erro máximo do dispositivo é aproximadamente $\pm 1,25 \mathrm{kPa}$, o que a princípio é um valor enorme para as medições realizadas, que foram todas abaixo de $2,85 \mathrm{kPa}$. Entretanto, como foi explicitado, utilizando um volume consideravel de dados adquiridos foi possivel mitigar drasticamente esse erro para valores em torno de $\pm 0,1 \mathrm{kPa}$ ou menores durante todo o experimento. Dessa forma, a utilização do sensor se mostrou pertinente na aquisição de dados nas condições utilizadas.

Outra opção, é a utilização de sensores com fundo de escala menor, como por exemplo o MPX5010, cuja a pressão máxima é de $10 \mathrm{kPa}$ e o erro máximo, segundo o fabricante, de aproximadamente $\pm 0,5 \mathrm{kPa}$.

Adicionalmente, o microcontrolador Arduino Uno® além de ser largamente utilizado em laboratórios acadêmicos, nos últimos anos tem ganhado espaço no meio industrial para automação de processos menos complexos. A aplicação estudada neste trabalho poderia ser empregada, por exemplo, em redes de distribuição de água, fazendo parte de um sistema de medição de vazão e controle de bombas e válvulas. 


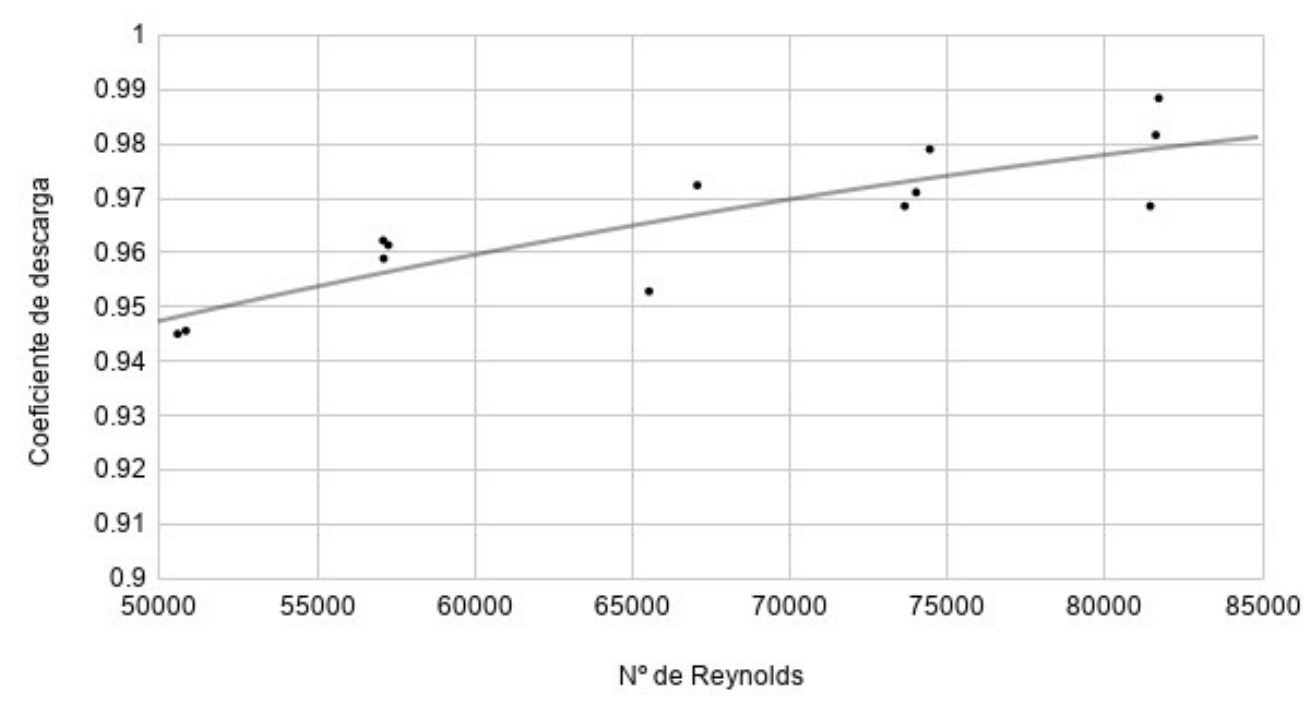

Figura 6 - Coeficiente de descarga obtido com o auxílio do manômetro em U.

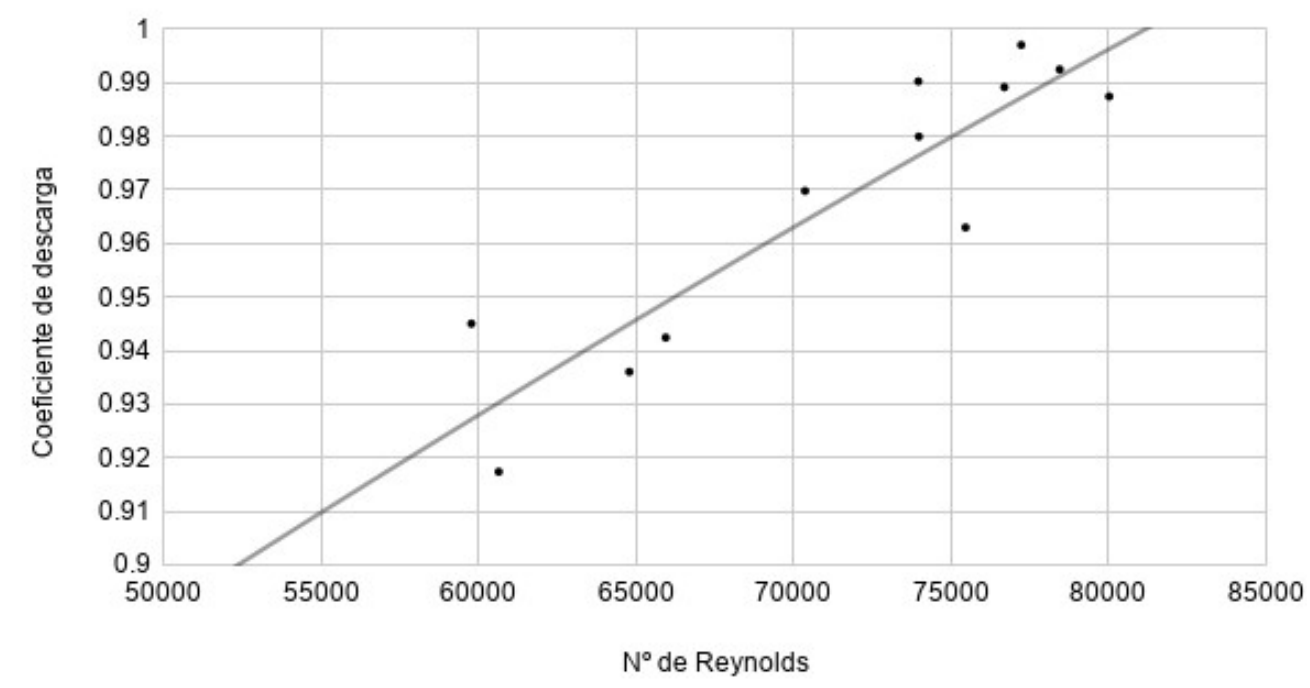

Figura 7 - Coeficiente de descarga obtida com o auxílio do sensor de diferença de pressão.

\section{CONCLUSÃO}

A determinação da curva do coeficiente de descarga realizada com o sensor de diferença de pressão demonstrou-se eficaz já que o erro resultante é tolerável para a maioria das aplicações de tubo Venturi, o que torna a descrição do coeficiente de novos tubos com sistemas semelhantes mais rápido e eficiente.

Esse estudo serve como base para pesquisas posteriores onde a proposta seria refinar o processo de aquisição de dados pelo MPX 5050, assim criando um método mais rápido e preciso para mensurar vazão utilizando uma montagem semelhante. Além disso, também possibilita que sejam implementados sistemas automatizados nos quais a vazão proveniente do sensor associado a microcontrolador e tubo de Venturi, seja um parâmetro central, como por exemplo nas redes municipais de distribuição de água, onde o conhecimento da vazão em tempo real é uma informação de extrema importância, inclusive para a tomada de decisões sobre o fluxo de bombeamento e de tratamento de água.

Os resultados experimentais deste estudo revelam que arranjos semelhantes ao utilizado podem resultar em leituras de vazão com grande confiabilidade, aliado a um custo de aquisição baixo. 
DEHKORDI, P. B.; COLOMBO, L. G. M.; GUILIZZONI, M.; SOTGIA, G. Simulation with experimental validation of oil-water core-annular flows through Venturi and Nozzle flow meters. Journal of Petroleum Science and Engineering, v.149, p. 540$552,2017$.

FARNELL. "DATASHEET MPX5050". Disponível em: $<$ farnell.com/datasheets/673750.pdf $>$ Acesso em: 17 set. 2017.

International Organization for Standardization. ISO 5167-4: Measurement of fluid flow by means of pressure differential devices inserted in circular crosssection conduits running full. Genebra, 2003.

LI, C.; MICKAN, B. Humidity effect on the calibration of discharge coefficients of critical flow Venturi nozzles in a pVTt facility. Flow Measurement and Instrumentation, v.46, Part A, p. 125-132, dez. 2015.

READER-HARRIS, M. J. Orifice Plates and Venturi Tubes. 1. ed. Glasgow: Springer, 2015.

TITHERADGE, P. J.; ROBERGS, R.; Venturi Tube Calibration For Airflow And Volume Measurement. Flow Measurement and Instrumentation, 2018 